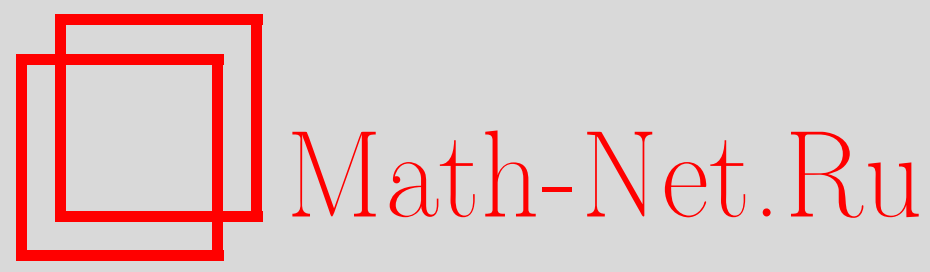

3. Х. Рахмонов, Тернарная задача Эстермана с почти равными слагаемыми, Матем. заметки, 2003, том 74, выпуск 4, 564-572

DOI: https://doi.org/10.4213/mzm291

Использование Общероссийского математического портала Math-Net.Ru подразумевает, что вы прочитали и согласны с пользовательским соглашением http://www.mathnet.ru/rus/agreement

Параметры загрузки:

IP: 18.207 .199 .55

26 апреля 2023 г., 04:05:59

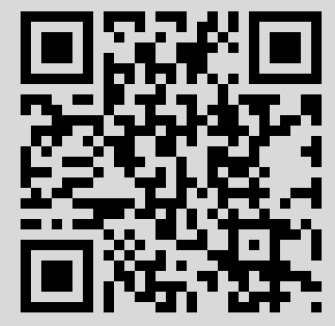


УДК 511

\section{ТЕРНАРНАЯ ЗАДАЧА ЭСТЕРМАНА \\ С ПОЧТИ РАВНЫМИ СЛАГАЕМЫМИ}

\section{3. Х. Рахмонов}

Доказана асимптотическая формула для количества представлений достаточно большого натурального числа $N$ в виде суммы двух простых и квадрата натурального, когда они почти равны.

Библиография: 6 названий.

1. Введение. Эстерман [1] доказал асимптотическую формулу для числа решений уравнения

$$
p_{1}+p_{2}+m^{2}=N,
$$

где $p_{1}, p_{2}-$ простые числа, $m$ - натуральное число.

Карацуба предложил исследовать эту задачу с более жесткими условиями, а именно, когда слагаемые почти равны. Теорема [2], [3] о поведении тригонометрических сумм с простьми числами, переменное суммирование которых принимает значения из коротких интервалов, в сочетании с оценками специальных тригонометрических сумм позволили доказать теорему Эстермана с почти равными слагаемьми.

Теорема. Пусть $N$ - достаточно большое натуральное число, $I(N, H)$ - число представлений $N$ суммой двух простых чисел $p_{1}, p_{2}$ и квадрата натурального $m$ с условиями

$$
\left|p_{i}-\frac{N}{3}\right| \leqslant H, \quad i=1,2, \quad\left|m^{2}-\frac{N}{3}\right| \leqslant H .
$$

Тогда при $H \geqslant N^{3 / 4} \mathscr{L}^{3}, \mathscr{L}=\ln N$, справедлива асимптотическая формула

$$
\begin{gathered}
I(N, H)=\frac{3 \sigma}{2} \frac{H^{2}}{\sqrt{N / 3} \mathscr{L}^{2}}+O\left(\frac{H^{2}}{\sqrt{N} \mathscr{L}^{3}}\right) \\
\sigma=\prod_{p}\left(1+\left(\frac{N}{p}\right) \frac{1}{(p-1)^{2}}\right), \quad\left(\frac{N}{p}\right)-\text { символ Лежандра. }
\end{gathered}
$$

\section{2. Вспомогательные утверждения.}

Лемма 1 [4]. Пусть действительная функиия $f(u)$ и монотонная функиия $g(u)$ удовлетворяют следующим условиям: $f^{\prime}(u)$ монотонна, $\left|f^{\prime}(u)\right| \geqslant m>0 u$ $|g(u)| \leqslant M$. Тогда справедлива оченка

$$
\int_{a}^{b} g(u) e(f(u)) d u \ll \frac{M}{m} .
$$


Лемма 2 [5]. Пусть $f(u)$ в интервале $(a, b)$ - вещественная дифференцируемая функиия, причем внутри интервала ее производная $f^{\prime}(u)$ монотонна и знакопостоянна и при постоянном $\delta$ таком, что $0<\delta<1$, удовлетворяет неравенству $f^{\prime}(u) \leqslant \delta$. Тогда имеем

$$
\sum_{a}^{b} e(f(n))=\int_{a}^{b} e(f(u)) d u+\theta\left(3+\frac{2 \delta}{1-\delta}\right)
$$

Лемма 3 [5]. Пусть $\alpha=a / q+\theta / q^{2},(a, q)=1, q \geqslant 1,|\theta| \leqslant 1$. Тогда при любом $\beta$, $U>0, P \geqslant 1$ имеем

$$
\sum_{n=1}^{P} \min \left(U, \frac{1}{\|\alpha n+\beta\|}\right) \leqslant 6\left(\frac{P}{q}+1\right)(U+q \ln q) .
$$

Пусть $N(\alpha, T, \chi)$ - число нулей $\rho=\beta+i \gamma$ функции $L(s, \chi)$ в области $\operatorname{Re} s \geqslant \alpha \geqslant 1 / 2$, $|\operatorname{Im} s| \leqslant T$. Если $\chi$ - главньй характер, тогда $N(\sigma, T, \chi)=N(\sigma, T)$ - число нулей функции Римана $\zeta(s)$ в указанной области.

ОПРЕДЕЛЕНИЕ. Пусть $c \geqslant 2, B \geqslant 1$ - абсолютные постоянные, $T \geqslant T_{0}>0$, $T^{\theta} H<T, \theta>0$. Тогда оценки вида

$$
\sum_{\chi \bmod q}[N(\alpha, T+H, \chi)-N(\alpha, T, \chi)] \ll(q T)^{c(1-\alpha)}(\ln q T)^{B}
$$

назьваются плотностным.ми теоремами в коротких прямоугольниках критической полосы.

Жан Тао [6] доказал, что если $H>T^{1 / 3}$, то соотношение (1) выполняется при $c=8 / 3$, $B=216$.

Приведем теорему, устанавливающую связь плотностных теорем для нулей L-рядов Дирихле в коротких прямоугольниках критической полосы с оценками линейных тригонометрических сумм с простыми числами, переменное суммирование которых принимает значение из коротких интервалов, т.е. с суммами вида

$$
S(\alpha ; x, y)=\sum_{x-y<n \leqslant x} \Lambda(n) e(\alpha n), \quad \alpha=\frac{a}{q}+\lambda, \quad(a, q)=1, \quad|\lambda| \leqslant \frac{1}{q \tau}, \quad 1 \leqslant q \leqslant \tau .
$$

Лемма 4 [3]. Пусть в сумме $S(\alpha ; x, y)$ выполнены оценки

$$
x \geqslant x_{0}>2, \quad y \geqslant h x^{(c-1) / c} \exp (\ln x)^{0.76}, \quad \tau \geqslant \frac{y^{2}}{x h}, \quad h \leqslant x^{1 / c}, \quad q \leqslant h .
$$

Тогда справедливо равенство

$$
S(\alpha ; x, y)=\frac{\mu(q)}{\phi(q)} \frac{\sin \pi \lambda y}{\pi \lambda} e\left(\lambda\left(x-\frac{y}{2}\right)\right)+O\left(\frac{y l^{B+3}}{q^{1 / 2}} F(q, x)\right),
$$

где $l=\ln x, b-$ произвольное фиксированное неотричательное число и

$$
F(q, x)= \begin{cases}\exp \left(-\ln ^{4} \ln x\right), & \text { если } q \leqslant l^{b}, \\ 1, & \text { если } q>l^{b} .\end{cases}
$$


Лемма 5. Пусть $\alpha=a / q+\lambda,(a, q)=1, q \leqslant \tau,|\lambda| \leqslant 1 /(q \tau), \tau \geqslant 20 y, y \leqslant x$, $\|t\|=\min (\{t\}, 1-\{t\}) u$

$$
m= \begin{cases}2 \lambda q x-\|2 \lambda q x\|, & \text { если }\{2 \lambda q x\} \leqslant 0.5, \\ 2 \lambda q x+\|2 \lambda q x\|, & \text { если }\{2 \lambda q x\} \geqslant 0.5 .\end{cases}
$$

Тогда имеет место соотношение

$$
T(\alpha ; x, y)=\sum_{x-y<n \leqslant x} e\left(\alpha n^{2}\right)=\frac{y}{q} \sum_{k=0}^{q-1} e\left(\frac{a k^{2}+m k}{q}\right) \gamma(\lambda ; x, y)+O(q),
$$

әде

$$
\gamma(\lambda ; x, y)=\int_{0}^{1} e\left(\lambda(x-u y)^{2}-\frac{m(x-u y)}{q}\right) d u .
$$

ДокаЗАТЕЛЬСтво. Имеем

$$
\begin{aligned}
T(\alpha ; x, y) & =\sum_{k=0}^{q-1} \sum_{x-y<q t+k \leqslant x} e\left(\left(\frac{a}{q}+\lambda\right)(q t+k)^{2}\right) \\
& =\sum_{k=0}^{q-1} e\left(\frac{a k^{2}}{q}\right) \sum_{\frac{x-y-k}{q}<t \leqslant \frac{x-k}{q}} e\left(\lambda(q t+k)^{2}\right) .
\end{aligned}
$$

Так как $m$ целое, то

$$
T(\alpha ; x, y)=\sum_{k=0}^{q-1} e\left(\frac{a k^{2}}{q}\right) \sum_{\frac{x-y-k}{q}<t \leqslant \frac{x-k}{q}} e\left(\lambda(q t+k)^{2}-m t\right) .
$$

Применяя лемму 2 при $f(t)=\lambda(q t+k)^{2}-m t$ и имея в виду, что

$$
\left|f^{\prime}(t)\right| \leqslant 2|\lambda| q(x-(q t+k))+0.5 \leqslant \frac{2 y}{\tau}+0.5 \leqslant 0.6,
$$

имеем

$$
\sum_{\frac{x-y-k}{q}<t \leqslant \frac{x-k}{q}} e\left(\lambda(q t+k)^{2}-m t\right)=\int_{\frac{x-y-k}{q}}^{\frac{x-k}{q}} e\left(\lambda(q t+k)^{2}-m t\right) d t+O(1) .
$$

Полагая $u=(x-k-q t) / y$, сделаем в последнем интеграле замену переменных, тогда

$$
\begin{aligned}
T(\alpha ; x, y) & =\sum_{k=0}^{q-1} e\left(\frac{a k^{2}}{q}\right) \frac{y}{q} \int_{0}^{1} e\left(\lambda(x-u y)^{2}-\frac{m(x-u y-k)}{q}\right) d u+O(q) \\
& =\frac{y}{q} \sum_{k=0}^{q-1} e\left(\frac{a k^{2}+m k}{q}\right) \gamma(\lambda ; x, y)+O(q) .
\end{aligned}
$$

Лемма доказана. 
3. Доказательство теоремы. Не ограничивая общности, будем считать, что $H=$ $2 N^{3 / 4} \mathscr{L}^{5 / 2}$. Пусть $Q=\mathscr{L}^{5}, \tau=H^{2} / N Q, \varkappa \tau=1, E=[-\varkappa, 1-\varkappa]$. Имеем

$$
\begin{gathered}
I(N, H)=\int_{E} S_{1}^{2}(\alpha ; N, H) T_{1}(\alpha ; N, H) e(-\alpha N) d \alpha \\
S_{1}(\alpha ; N, H)=\sum_{|p-N / 3| \leqslant H} e(\alpha p) ; \quad T_{1}(\alpha ; N, H)=\sum_{\left|n^{2}-N / 3\right| \leqslant H} e\left(\alpha n^{2}\right) .
\end{gathered}
$$

Согласно теореме Дирихле о приближении действительных чисел рациональными числами, каждое $\alpha$ из промежутка $E$ представим в виде

$$
\alpha=\frac{a}{q}+\lambda, \quad(a, q)=1, \quad 1 \leqslant q \leqslant \tau, \quad|\lambda| \leqslant \frac{1}{(q \tau)} .
$$

Легко видеть, что в этом представлении $0 \leqslant a \leqslant q-1$, причем $a=0$ лишь при $q=1$. Через $E_{1}$ обозначим множество тех $\alpha$, для которых $q \leqslant Q$ в представлении (2), через $E_{2}$ обозначим оставшиеся $\alpha$. Множество $E_{1}$ состоит из непересекающихся отрезков. Разобьем множество $E_{1}$ на множества $E_{11}$ и $E_{12}$ следуюшим образом:

$$
E_{11}=\left\{\alpha: \alpha \in E_{1},\left|\alpha-\frac{a}{q}\right| \leqslant \frac{\mathscr{L}^{2}}{H}\right\}, \quad E_{12}=\left\{\alpha: \alpha \in E_{1}, \frac{\mathscr{L}^{2}}{H}<\left|\alpha-\frac{a}{q}\right| \leqslant \frac{1}{q \tau}\right\} .
$$

Обозначим через $I_{11}, I_{12}$ и $I_{2}$ соответственно интегралы по множествам $E_{11}, E_{12}$ и $E_{2}$. Имеем

$$
I(N, H)=I_{11}+I_{12}+I_{2} .
$$

В последней формуле первый член, т.е. $I_{11}$ доставляет главньй член асимптотической формулы для $I(N, H)$, а $I_{12}$ и $I_{2}$ входят в его остаточный член.

Вычисление интеграла $I_{11}$. По определению интеграла $I_{11}$ имеем

$$
\begin{gathered}
I_{11}=\int_{E_{11}} S_{1}^{2}(\alpha ; N, H) T_{1}(\alpha ; N, H) e(-\alpha N) d \alpha=\sum_{q \leqslant Q} \sum_{\substack{a=0 \\
(a, q)=1}}^{q-1} I(a, q), \\
I(a, q)=\int_{|\lambda| \leqslant \mathscr{L}^{2} / H} S_{1}^{2}\left(\frac{a}{q}+\lambda ; N, H\right) T_{1}\left(\frac{a}{q}+\lambda ; N, H\right) e\left(-\left(\frac{a}{q}+\lambda\right) N\right) d \lambda .
\end{gathered}
$$

Выражая сумму $S_{1}(\alpha ; N, H)$ через сумму $S(\alpha ; x, y)$ и пользуясь леммой 4 , находим

$$
\begin{aligned}
S_{1}\left(\frac{a}{q}+\lambda ; N, H\right) & =\left(\ln \left(\frac{N}{3}\right)\right)^{-1} S\left(\frac{a}{q}+\lambda ; \frac{N}{3}+H, 2 H\right)+O\left(H^{2} N^{-1} \mathscr{L}^{-1}\right) \\
& =\left(\ln \left(\frac{N}{3}\right)\right)^{-1} \frac{\mu(q)}{\varphi(q)} \frac{\sin 2 \pi \lambda H}{\pi \lambda} e\left(\frac{\lambda N}{3}\right)+O\left(H \exp \left(-\ln ^{4} \mathscr{L}\right)\right) .
\end{aligned}
$$

Возводя обе части получившейся формулы в квадрат и имея в виду, что

$$
\frac{\mu(q)}{\varphi(q)} \frac{\sin 2 \pi \lambda H}{\pi \lambda} e\left(\frac{\lambda N}{3}\right) \leqslant H,
$$


найдем

$$
S_{1}^{2}\left(\frac{a}{q}+\lambda ; N, H\right)=\left(\ln \left(\frac{N}{3}\right)\right)^{-2} \frac{\mu^{2}(q)}{\varphi^{2}(q)} \frac{\sin ^{2} 2 \pi \lambda H}{(\pi \lambda)^{2}} e\left(\frac{2 \lambda N}{3}\right)+O\left(H^{2} \exp \left(-\ln ^{4} \mathscr{L}\right)\right) .
$$

Пусть

$$
T(\alpha ; x, y)=\sum_{x-y<n \leqslant x} e\left(\alpha n^{2}\right)
$$

Пользуясь соотношением

$$
\sqrt{\frac{N}{3} \pm H}=N_{1} \pm H_{1}+O\left(H^{2} N^{-3 / 2}\right), \quad N_{1}=\sqrt{\frac{N}{3}}, \quad H_{1}=\frac{H}{2 \sqrt{N / 3}}
$$

для $T_{1}(\alpha ; N, H)$ имеем

$$
\begin{aligned}
T_{1}(\alpha ; N, H) & =T\left(\alpha ; \sqrt{\frac{N}{3}+H}, \sqrt{\frac{N}{3}+H}-\sqrt{\frac{N}{3}-H}\right) \\
& =T\left(\alpha ; N_{1}+H_{1}, 2 H_{1}\right)+O\left(H^{2} N^{-3 / 2}\right) .
\end{aligned}
$$

Применяя к сумме $T\left(\alpha ; N_{1}+H_{1}, 2 H_{1}\right)$ лемму 5 и имея в виду, что

$$
m=2 \lambda q\left(N_{1}+H_{1}\right) \pm\left\|2 \lambda q\left(N_{1}+H_{1}\right)\right\|=0
$$

найдем

$$
T_{1}\left(\frac{a}{q}+\lambda ; N, H\right)=\frac{H}{q \sqrt{N / 3}} S(a, q) \gamma\left(\lambda ; N_{1}+H_{1}, 2 H_{1}\right)+O(q)+O\left(H^{2} N^{-3 / 2}\right)
$$

где

$$
\gamma(\lambda ; x, y)=\int_{0}^{1} e\left(\lambda(x-u y)^{2}\right) d u, \quad S(a, q)=\sum_{k=0}^{q-1} e\left(\frac{a k^{2}}{q}\right)-\text { сумма Гаусса. }
$$

Тем самьм, для $I(a, q)$ находим

$$
I(a, q)=\left(\ln \left(\frac{N}{3}\right)\right)^{-2} \frac{H}{\sqrt{N / 3}} \frac{\mu^{2}(q)}{q \varphi^{2}(q)} S(a, q) e\left(-\frac{a N}{q}\right) \int_{0}^{1} F(t) d t+R_{1}
$$

где

$$
\begin{aligned}
F(t) & =\int_{|\lambda| \leqslant \mathscr{L}^{2} / H} \frac{\sin ^{2} 2 \pi \lambda H}{(\pi \lambda)^{2}} e\left(\lambda\left(\frac{2 N}{3}+\left(\sqrt{N / 3}+\frac{H}{2 \sqrt{N / 3}}-t \frac{H}{\sqrt{N / 3}}\right)^{2}-N\right)\right) d \lambda, \\
R_{1} & \ll\left[S_{1}^{2}(\alpha ; N, H)\left(q+H^{2} N^{-3 / 2}\right)+H q^{-1} N^{-1 / 2} S(a, q) H^{2} \exp \left(-\ln ^{4} \mathscr{L}\right)\right] H^{-1} \mathscr{L}^{2} \\
& \ll\left[H^{2} \mathscr{L}^{-2}\left(q+H^{2} N^{-3 / 2}\right)+H N^{-1 / 2} q^{-1 / 2} H^{2} \exp \left(-\ln ^{4} \mathscr{L}\right)\right] H^{-1} \mathscr{L}^{2} \\
& \ll H Q+H^{3} N^{-3 / 2}+H^{2} N^{-1 / 2} \mathscr{L}^{2} \exp \left(-\ln ^{4} \mathscr{L}\right) \ll H^{2} N^{-1 / 2} \mathscr{L}^{-3} Q^{-3}
\end{aligned}
$$


В интеграле $F(t)$ сделаем замену переменных и получим

$$
F(t)=\frac{2 H}{\pi} \int_{|u| \leqslant 2 \pi \mathscr{L}^{2}} \frac{\sin ^{2} u}{u^{2}} e\left(\frac{u}{2 \pi}(1-2 t)\right) e\left(\frac{3 H u}{8 \pi N}(1-2 t)^{2}\right) d u .
$$

Пользуясь соотношением

$$
e\left(\frac{3 H u}{8 \pi N}(1-2 t)^{2}\right)=1+O\left(\frac{u(1-2 t) H}{N}\right)=1+O\left(\frac{u H}{N}\right)
$$

находим

$$
F(t)=\frac{2 H}{\pi} \int_{|u| \leqslant 2 \pi \mathscr{L}^{2}} \frac{\sin ^{2} u}{u^{2}} e\left(\frac{u}{2 \pi}(1-2 t)\right) d u+O\left(\frac{H^{2} \mathscr{L}}{N}\right) .
$$

Интегрируем обе части этого равенства по $t$ от 0 до 1 , затем интеграл по $u$ заменим близким к нему несобственным интегралом, не зависяшим от $\mathscr{L}$. Далее, пользуясь соотношениями

$$
\int_{0}^{\infty} \frac{\sin ^{3} u}{u^{3}} d u=\frac{3 \pi}{8}, \quad \int_{2 \pi \mathscr{L}^{2}}^{\infty} \frac{\sin ^{3} u}{u^{3}} d u \ll \mathscr{L}^{-6},
$$

найдем

$$
\int_{0}^{1} F(t) d t=\frac{4 H}{\pi}\left(\int_{0}^{\infty} \frac{\sin ^{3} u}{u^{3}} d u-\int_{2 \pi \mathscr{L}^{2}}^{\infty} \frac{\sin ^{3} u}{u^{3}} d u\right)+O\left(\frac{H^{2} \mathscr{L}}{N}\right)=\frac{3 H}{2}+O\left(\frac{H}{\mathscr{L}^{6}}\right) .
$$

Подставив это в формулу для $I(a, q)$, найдем

$$
I(a, q)=\frac{3 H^{2}}{2 \sqrt{N / 3} \ln ^{2}(N / 3)} \frac{\mu^{2}(q) S(a, q)}{q \varphi^{2}(q)} e(-a N / q)+O\left(\frac{H^{2}}{\sqrt{N} \mathscr{L}^{3}}\left(\frac{1}{\sqrt{q} \varphi^{2}(q)}+\frac{1}{Q^{3}}\right)\right) .
$$

Следовательно,

$$
I_{11}=\frac{3 H^{2}}{2 \sqrt{N / 3} \ln ^{2}(N / 3)} \sum_{q \leqslant Q} \frac{\mu^{2}(q)}{q \varphi^{2}(q)} \sum_{\substack{a=0 \\(a, q)=1}}^{q} S(a, q) e(-a N / q)+O\left(\frac{H^{2}}{\sqrt{N} \mathscr{L}^{3}}\right) .
$$

Двойную сумму в последнем равенстве заменим близким к ней бесконечным рядом, не зависяшим от $Q$, т.е.

$$
\begin{gathered}
\sum_{q \leqslant Q} \frac{\mu^{2}(q)}{q \varphi^{2}(q)} \sum_{\substack{a=1 \\
(a, q)=1}}^{q} S(a, q) e\left(-\frac{a N}{q}\right)=\sigma(N)+R(N), \\
\sigma(N)=\sum_{q=1}^{\infty} \frac{\mu^{2}(q)}{q \varphi^{2}(q)} \sum_{\substack{a=1 \\
(a, q)=1}}^{q} S(a, q) e\left(-\frac{a N}{q}\right), \\
|R(N)|=\left|\sum_{q>Q} \frac{\mu^{2}(q)}{q \varphi^{2}(q)} \sum_{\substack{a=1 \\
(a, q)=1}}^{q} S(a, q) e\left(-\frac{a N}{q}\right)\right| \\
\leqslant \sum_{q>Q} \frac{\mu^{2}(q)}{\sqrt{q} \varphi(q)} \ll \sum_{q>Q} \frac{\ln \ln q}{q^{3 / 2}} \ll Q^{-1 / 2+\varepsilon} .
\end{gathered}
$$


Имеем

$$
\sigma(N)=\sum_{q=1}^{\infty} \frac{\mu^{2}(q)}{q \varphi^{2}(q)} \Phi(q), \quad \Phi(q)=\sum_{\substack{a=1 \\(a, q)=1}}^{q} S(a, q) e\left(-\frac{a N}{q}\right)
$$

Если $q$ - четное безквадратное число и $(a, q)=1$, то $S(a, q)=0$. Действительно, если $q=2 p_{1} p_{2} \cdots p_{k}$, то полагая $q_{0}=q 2^{-1}, q_{i}=q p_{i}^{-1}, i=\overline{1, n}$, представив $a$ в виде $a=a_{0} q_{0}+a_{1} q_{1}+\cdots+a_{k} q_{k}$ и имея в виду, что $S\left(a_{0} q_{0}, 2\right)=0$, получаем

$$
S(a, q)=S\left(a q_{0}, 2\right) S\left(a q_{1}, p_{1}\right) \cdots S\left(a q_{k}, p_{k}\right)=S\left(a_{0}, 2\right) S\left(a_{1}, p_{1}\right) \cdots S\left(a_{k}, p_{k}\right)=0 .
$$

Таким образом, не ограничивая общности, будем считать, что $q$ - нечетное безквадратное число, т.е. $q=p_{1} p_{2} \cdots p_{k}$. Пользуясь мультипликативностью $\Phi(q)$ и формулой, которая устанавливает связь между значениями символов Лежандра и значениями сумм Гаусса, находим

$$
\Phi(q)=\prod_{i=1}^{k} \sum_{a_{i}=1}^{p_{i}-1} S\left(a_{i}, p_{i}\right) e\left(-\frac{a_{i} N}{p_{i}}\right)=\prod_{i=1}^{k} S\left(1, p_{i}\right) \sum_{a_{i}=1}^{p_{i}-1}\left(\frac{a_{i}}{p_{i}}\right) e\left(-\frac{a_{i} N}{p_{i}}\right) .
$$

Если $(N, q)>1$, то существует по крайней мере один $p_{i}$ такой, что $N \equiv 0\left(\bmod p_{i}\right)$. Поэтому, при $(N, q)>1$, имеем

$$
\Phi(q)=\Phi\left(q p_{i}^{-1}\right) \Phi\left(p_{i}\right)=\Phi\left(q p_{i}^{-1}\right) S\left(1, p_{i}\right) \sum_{a_{i}=1}^{p_{i}-1}\left(\frac{a_{i}}{p_{i}}\right)=0
$$

Если $(N, q)=1$, то

$$
\Phi(q)=\prod_{i=1}^{k} S\left(1, p_{i}\right)\left(\frac{-N}{p_{i}}\right) \sum_{a_{i}=1}^{p_{i}-1}\left(\frac{a_{i}}{p_{i}}\right) e\left(-\frac{a_{i}}{p_{i}}\right)=\prod_{i=1}^{k}\left(\frac{-N}{p_{i}}\right) S^{2}\left(1, p_{i}\right)=\left(\frac{-N}{q}\right) S^{2}(1, q),
$$

где

$$
\prod_{i=1}^{k}\left(\frac{-N}{p_{i}}\right)=\left(\frac{-N}{q}\right) \text { - символ Якоби. }
$$

Следовательно,

$$
\sigma(N)=\sum_{q=1}^{\infty} \frac{\mu^{2}(q)}{q \varphi^{2}(q)} \Phi(q)=\prod_{p}\left(1+\frac{1}{p(p-1)^{2}}\left(\frac{-N}{p}\right) S^{2}(1, p)\right)
$$

Пользуясь соотношением $(p \neq 2)$

$$
\left(\frac{-1}{p}\right) S^{2}(1, p)=p
$$

найдем

$$
\sigma(N)=\prod_{p}\left(1+\left(\frac{N}{p}\right) \frac{1}{(p-1)^{2}}\right)
$$


Таким образом, получаем

$$
\begin{aligned}
I_{11} & =\frac{3 \sigma(N) H^{2}}{2 \sqrt{N / 3} \ln ^{2}(N / 3)}+O\left(\frac{H^{2}}{\sqrt{N} \mathscr{L}^{3}}+H Q^{3} \mathscr{L}+\frac{H^{3} Q^{2} \mathscr{L}}{N^{3 / 2}}+\frac{H^{2}}{\sqrt{N} \mathscr{L}^{2}} Q^{-1 / 2+\varepsilon}\right) \\
& =\frac{3 \sigma(N) H^{2}}{2 \sqrt{N / 3} \mathscr{L}^{2}}+O\left(\frac{H^{2}}{\sqrt{N} \mathscr{L}^{3}}\right) .
\end{aligned}
$$

Оценка интеграла $I_{2}$. Оценим $T_{1}(\alpha ; N, H)$ для $\alpha$ из множества $E_{2}$. Если $\alpha \in E_{2}$, то

$$
\alpha=\frac{a}{q}+\lambda, \quad(a, q)=1, \quad Q \leqslant q \leqslant \tau, \quad|\lambda| \leqslant \frac{1}{(q \tau)} .
$$

Согласно соотношению (3), имеем

$$
\begin{gathered}
T_{1}(\alpha ; N, H) \ll\left|T\left(\alpha, N_{1}+H_{1}, 2 H_{1}\right)\right|+H N^{-1 / 2} \mathscr{L}^{-2}, \\
N_{1}=\sqrt{\frac{N}{3}}, \quad H_{1}=\frac{H}{2 \sqrt{N / 3}} .
\end{gathered}
$$

Так как

$$
\begin{aligned}
& \left|T\left(\alpha ; N_{1}+H_{1}, 2 H_{1}\right)\right|^{2}=\sum_{N_{1}-H_{1}<m, n \leqslant N_{1}+H_{1}} e\left(\alpha\left(m^{2}-n^{2}\right)\right) \\
& \quad=\sum_{n_{1}-H_{1}<n \leqslant N_{1}+H_{1}} e(\alpha k(k+2 n)) \\
& \quad \leqslant\left.\sum_{|k| \leqslant 2 H_{1}}\right|_{N_{1}-H_{1}-n<k \leqslant H_{1}+H_{1}-k<n \leqslant N_{1}+H_{1}-k} e(2 \alpha k n) \mid \\
& \quad \leqslant \sum_{|k| \leqslant 2 H_{1}} \min \left(2 H_{1}, \frac{1}{|\sin 2 \pi \alpha k|}\right) \leqslant \sum_{|k| \leqslant 2 H_{1}} \min \left(2 H_{1}, \frac{1}{2|| 2 \alpha k||}\right),
\end{aligned}
$$

то применив к последней сумме лемму 3 , найдем

$$
|T(\alpha ; x, y)|^{2} \ll\left(\frac{H}{q \sqrt{N}}+1\right)\left(\frac{H}{\sqrt{N}}+q \ln q\right) \ll\left(\frac{H^{2}}{Q N}+\frac{H}{\sqrt{N}}+\tau\right) \mathscr{L} .
$$

Подставляя найденное выражение в (4) и пользуясь значениями $Q$ и $\tau$, находим

$$
T_{1}(\alpha, N, H) \ll \frac{H}{\sqrt{N}} \mathscr{L}^{-2} .
$$

Следовательно,

$$
I_{2} \ll \max _{\alpha \in E_{2}}\left|T_{1}(\alpha, N, H)\right| \int_{0}^{1}\left|S_{1}(\alpha ; N, H)\right|^{2} d \alpha \ll H^{2} N^{-1 / 2} \mathscr{L}^{-3} .
$$


Оценка интеграла $I_{12}$. Если $\alpha \in E_{12}$, то

$$
\alpha=\frac{a}{q}+\lambda, \quad(a, q)=1, \quad \frac{\mathscr{L}^{3}}{H}<|\lambda| \leqslant \frac{1}{(q \tau)}, \quad 1 \leqslant q \leqslant Q .
$$

Оценим $T_{1}(\alpha ; N, H)$. Согласно соотношению (4) и лемме 5 , имея в виду, что если $\alpha \in E_{12}$ и $H=2 N^{3 / 4} \mathscr{L}^{5 / 2}$, то $m=2 \lambda q\left(N_{1}+H_{1}\right) \pm\left\|2 \lambda q\left(N_{1}+H_{1}\right)\right\|=0$, найдем

$$
T_{1}\left(\frac{a}{q}+\lambda ; N, H\right) \ll \frac{H}{q \sqrt{N}}\left|S(a, q) \gamma\left(\lambda ; N_{1}+H_{1}, 2 H_{1}\right)\right|+H N^{-1 / 2} \mathscr{L}^{-2},
$$

где

$$
\gamma\left(\lambda ; N_{1}+H_{1}, 2 H_{1}\right)=\int_{0}^{1} e\left(\lambda\left(N_{1}+H_{1}-2 H_{1} u\right)^{2}\right) d u .
$$

Применяя к оценке интеграла $\gamma\left(\lambda ; N_{1}+H_{1}, 2 H_{1}\right)$ лемму 1 , полагая $f(t)=\lambda\left(N_{1}+H_{1}-\right.$ $\left.2 H_{1} t\right)^{2}$ и имея в виду, что $f^{\prime}(t) \geqslant \mathscr{L}^{2}$, находим

$$
T_{1}\left(\frac{a}{q}+\lambda ; N, H\right) \ll H N^{-1 / 2} \mathscr{L}^{-2} .
$$

Таким образом,

$$
I_{12} \ll \max _{\alpha \in E_{12}}\left|T_{1}(\alpha, N, H)\right| \int_{0}^{1}\left|S_{1}(\alpha ; N, H)\right|^{2} d \alpha \ll H^{2} N^{-1 / 2} \mathscr{L}^{-3} .
$$

Теорема доказана.

\section{СПИСОК ЦИТИРОВАННОЙ ЛИТЕРАТУРЫ}

[1] Estermann T. Proof that every large integer is the sum of two primes and square // Proc. London Math. Soc. 1937. V. 11. P. 501-516.

[2] Рахмонов 3. Х. Средние значения функции Чебышева в коротких интервалах // Тр. международной конференции "Современные проблемы математики и механики" посвященной 175-летию П. Л. Чебьшева. М.: МГУ, 1996. С. 125-189.

[3] Рахмонов 3. Х. Короткие линейные тригонометрические суммы с простыми числами // Докл. АН РТ. 2000. Т. 43. №3.

[4] Архипов Г. И., Карацуба А. А., Чубариков В. Н. Теория кратных тригонометрических сумм. М.: Наука, 1987.

[5] Виноградов И. М. Метод тригонометрических сумм в теории чисел. М.: Наука, 1976.

[6] Zhan Tao. On represention of a large odd integer as a sum of three almost primes // Acta Math. Sinica. 1991. V. 7. № 3. P. 259-272. 